\title{
Digital Geometry for Image-Based Metrology
}

\author{
Alfred M. Bruckstein \\ Ollendorff Professor of Science \\ Computer Science Department Technion, IIT 32000, Haifa, Israel \\ freddy@cs.technion.ac.il
}

\begin{abstract}
This paper surveys several interesting issues arising in digital geometry due to the need to perform accurate automated measurements on objects that are seen through the eyes of various types of imaging devices. These devices are typically regular arrays of (light) sensors and provide us matrices of quantized probings of the objects being looked at. In this setting, the natural questions that may be posed are: how accurately can we locate and recognize these objects from classes of possible objects, and how precisely can we measure various geometric properties of the objects of interest, how accurately can we locate them given the limitations imposed upon us by the geometry of the sensor lattices and the quantization and noise omnipresent in the sensor device output. Yet another exciting area of investigation is the design of (classes of) objects that enable optimal exploitation of the imaging device capabilities, in the sense of yielding the most accurate measurements possible.
\end{abstract}

\section{Introduction}

Scanned character recognition systems are working quite well by now, several companies have grown based on the need to do image based inspection for quality control in the semiconductor industry and, in general, automated visual inspection is by now widely used in many areas of manufacturing. In these important applications one needs to perform a series of precise geometric measurements based on images of various types of (planar) objects or shapes. The images of these shapes are provided by sensors with limited capabilities. These sensors are spatially arranged in (regular) planar arrays providing (matrices of) quantized (pixel-)values that need to be processed by automated metrology systems to extract information on the location, identity, size and orientation, texture and color, of the objects being looked at. The geometry of the sensor array is a crucial factor in the measurement performances that are possible. When sensor arrays are regular planar grids, we have to deal with a wealth of issues involving geometry on the integer grid. This is how digital geometry enters the picture in industrial metrology tasks, in very fundamental ways.

\section{The Digitization Model and the Metrology Tasks}

We shall here assume that the planar shapes, the objects we are interested to locate, measure and recognize are binary (black on a white background) and live 
in the real plane, $\mathbf{R}$. Hence their full description can be given via an indicator function $\xi(x, y)$ which is 1 (black) on the shape and 0 (white) on the background. The digitization process will be by point sampling on the integer grid, $\mathbf{Z}^{2}$, hence the result of digitization will be a discrete indicator function on the integer grid: a discrete binary image, or matrix of picture elements, or pixels. The generic problem we shall deal with is: given the discretized shape recover as much information as possible on the "pre-image", i.e. on the original binary shape that lives on the continuous real plane. The necessary information on the pre-image shape might be its location and orientation area, perimeter, etc. In order to solve the particular problem at hand we shall also exploit whatever prior information we may have on the continuous pre-images. This prior information sometimes defines the objects or shapes we digitize as members of parameterized sets of possible pre-images. For example, we might know that the shapes we are called upon to measure are circular with varying locations and sizes. In this case the parameter defining the particular object instance being analyzed from its digitization is a vector comprising three numbers: two coordinates pointing out the center of the disk and a positive number providing its radius.

\section{The Wonders and Uses of Digital Lines}

Digital lines, or digital straight segments result from sampling half-plane preimages or planar polygonal shapes (on the integer grid). More is known about this topic than anyone can possibly know, but I would dare to say that the basic facts are both simple and beautiful. Half-planes are not very interesting or practically useful objects, however they already pose the following metrology problem: given the digital image of a half-plane, locate it as precisely as possible. Of course, we must ask ourselves whether and how our location estimation improves as we see more and more of the digitized boundary. Well, it turns out that we can think about the location estimation problem as a problem of determining the halfplane pre-images that satisfy all the constraints that the digitized image provides. Indeed every grid-point pixel that is 0 (white) will tell us that the half-plane does not cover that location while every black (1) pixel will indicate that the halfplane covers its position. It should come as no surprise that the boundary pixels, i.e. the locations where white pixels are neighboring black ones, carry all the information. The constraint that a certain location in the plane belongs, or does not belong to the half-plane that is being probed translates into a condition that the boundary line has a slope and intercept pair in a half-plane defined in the dual representation space (which is called in pattern recognition circles the Hough parameter plane). Therefore, as we collect progressively more data in the "imageplane" we have to intersect more an more half-planes in the Hough plane to get the so called "locale", or the uncertainty region in parameter space where the boundary line parameters lie, see [8]. Looking at the grid geometry and analyzing the lines that correspond to grid-points in the dual plane one quickly realizes that only the boundary points contribute to setting the limits of the locale of interest, and a careful analysis reveals that, due to the regularity of the sampling grid, 
the locales are always polygons of at most four sides, see [6]. Hence as more and more consecutive boundary points are added to the pool of information on the digitized half plane, we have to perform half-plane intersections with at most four sided polygonal locales to update them. Clearly the locales generally decrease in size as the number of points increases, and we can get exact estimates on the uncertainty behavior as the jagged boundary is progressively revealed. This idea, combining Leo Dorst's discovery on the geometry of locales for digital straight lines with the process of successively performing the half-plane intersections for each new data point while walking along the jagged digitized boundary, led to the simplest, and only recursive $\mathrm{O}$ (length) algorithm for detecting straight edge segments. For a complete description of this algorithm see [13].

The jagged edges that result from discretizing half-planes have a beautiful, self-similar structure, intimately related to the structure of the real number that defines the slope of their boundary line. One can readily see that at various sampling resolutions the boundary maintains its jaggedness in a fractal manner, but here we mean a different type of self-similarity, inherent in the jagged boundaries at any given resolution! The paper [3] summarizes the wealth of interesting and beautiful properties that were described over many years of research on digital straight lines using a very simple unifying principle: invariance under re-encoding with respect to regular grids embedded into the integer lattice. Not only does this principle help in deriving in a very straightforward manner digital straight edge properties that were discovered and proved in sinuous ways, but it also points out all the selfsimilarity type properties that are possible, making nice connections to number-theoretic issues that arise in this context and the general linear group $G L(2, Z)$ that describes all integer lattice isomorphisms.

Using the wonderful properties of digital straight lines, we can not only solve the above-mentioned, and somewhat theoretical issue of locating a half-plane object of infinite extent but we can also address some very practical issues like measuring the perimeters of general planar shapes from their versions digitized on regular grids of pixels. Indeed, analyzing the properties of digitized lines made possible the rational design of some very simple and accurate perimeter estimators, based on classifications of the boundary pixels into different classes according to the jaggedness of their neighborhoods. Building upon earlier work of Proffit and Rosen [16], Koplowitz and Bruckstein proposed a general methodology for the design of simple and accurate perimeter estimation algorithms that are based on minimizing the maximum error that would be incurred for digitized straight edges over all orientations [11]. This methodology enables predictions of the expected performance for shapes having arbitrary, but bounded curvature, boundaries. Note also that using the recursive $\mathrm{O}(1)$-per boundary pixel algorithm for detecting digital straightness of Lindenbaum and Bruckstein [13] one could parse general, curved object boundaries into digitally straight segments and then estimate the pre-image object's perimeter as a sum of the lengths of the line-segments so-detected. In terms of the methodology presented in [13] this algorithms yields zero error for digital straight edges of infinite extent at all orientations, and hence should be the best perimeter estimator ever obtainable! 


\section{Digital Disks, Convex and Star-Shaped Objects}

From the realm of half-plane objects we can move to either infinite extent regions that have more complex boundaries (say parabolas, hyperbolae or some periodic functions along a principal direction) or to the analysis of finite extent objects like polygons, disks and other interesting shapes. Some work has indeed been done on detecting polygonal preimages from their digitized versions, and in fact a good algorithm for parsing a jagged boundary into digital straight segments turns out to be a crucial ingredient in solving various issues regarding the metrology of such objects.

Suppose next that we have the prior information that the objects discretized are disks of various locations and sizes. Then the metrology question arising naturally is: how precisely can we determine the location of a disk and its radius. Considering the digitization model by point sampling, as discussed above, given a digitized image of black and white pixels, we know that if a certain point in the plane is the center of a disk of unknown radius, this point will necessarily be closer to all black grid points than to any white grid point. Hence the locus of all possible points in the plane closer to all black points than to any white points is the locale of possible disk centers, and its size will quantify our uncertainty in locating the object in the preimage plane. It is interesting to note that this locale can be found without knowledge on the radius, which will still need to be estimated. It turns out that the locale as defined above is a well-known concept in computational geometry, and it is known that it is a convex region in the plane. Efrat and Gotsman have done a careful analysis of the problem and produced an $O(R \log R)$ algorithm to determine the locale, where $R$ is the radius of the disk. We refer the interested reader to the paper [7] for details. Note again that the locale we are talking about is independent of the radius parameter. Had we prior knowledge on the exact radius, the location of the disk center could be determined by intersecting all disks of radius $R$ around the black grid points with all the complements of disks or radius $R$ around the white (uncovered) grid points. The resulting intersection locale is generally not a convex shape, due to the precise knowledge of the radius.

For general convex shapes the question of determining the location and area and perimeter cannot be addressed in any generality. The digitized version of a convex shape is a set of black grid points on a background of white ones. As a union of square pixels the digitized shape will not be convex. Hence much work was done addressing the question whether there is a good definition of convexity for discrete objects [18]. A variety of proposals were made and can be found in the literature. The metrology questions however, in all cases remain: determine with best precision the location (first order moments), orientation (second order moments) and other metric properties, like area (zeroth order moment) and perimeter of the shape. These questions, too have received some attention. It turns out that computing the moments of the black grid points yields good estimates for the corresponding continuous quantities, and more refined, boundary estimation procedures (say, based on polygonalization of the jagged boundary via an efficient digital straight segment detection, as discussed 
above) do indeed provide improved estimates but the improvement needs to be carefully weighed against the increased complexity involved.

Among the many procedures that propose polygonal approximations to preimages based on the discrete grid points that were covered by the shape, and also based on the ones that were not covered, one stands out in elegance and usefulness: the minimum perimeter polygon that is enclosing all black (covered) points and excludes all white (uncovered) ones. This minimum perimeter polygon turns out to be the relative convex hull of the black points with respect to the white ones. It can be computed easily and may serve as a good approximation for preimages for all metrology purposes.

So far we talked about disks and convex objects. The next level of complexity in planar shapes are the so called star-shaped objects. These are defined as the shapes that have a "kernel region" inside them so that from any point in the kernel the entire boundary of the shape can be "seen", i.e. a line from the chosen point to any boundary point will lie entirely inside the shape. It is easy to see that this definition generalizes convexity an a rather natural way and that the kernels must be convex regions. Determining star-shapedness of a planar shape is not a too difficult task for polygons and for spline-gons and the algorithms for doing this rely on locating and using the inflection points on the boundary, and intersecting the regions in the plane from where the convex boundary regions are seen, see [1]. As with the notion of convexity, determining digital star-shapedness posed a series of special problems that needed careful analysis. This was the topic of a paper by Shaked, Koplowitz and Bruckstein, and there is was shown that the relative convex hull, or minimal perimeter polygon of the grid points covered by the shape wuth respect to the ones that remained uncovered, provides a convenient computational way to determine digital star-shapedness, see [17].

\section{Shape Designs for Good Metrology}

Up to this point we have discussed ways to analyze and measure planar shapes when seen through the looking glass of grid probing, or point-sampling discretization. The classes of shapes were assumed given in some perhaps parameterized form, and we dealt with questions about recovering their various features and parameters, or about measuring their size and perimeter and determining their location with the highest precision possible.

When considering such issues, a further question that can be posed is the following: design planar shapes or collections of shapes that will interact with the discretization process in such a way that the quantities we need to measure will be very easily read out in the discretized images we get. Could we design an object in the plane (that can be a union of continuous binary shapes), so that digitization of this object translated to various locations, will yield black and white patterns on the (discretization) grid that clearly exhibit, say in a binary representation, the $X$ and $Y$ translation values up to a certain desired precision?

Interestingly, recently a new pen-like device was invented and advertised, that has the following feature: it automatically computes with very high precision the 
location of its tip on any of the pages of a paper pad by looking at a faint pattern of dots that is printed on these sheets of paper. The pattern of these dots is so designed that the image obtained on any small region as seen by the pen near it's tip (with the help of a tiny light detector array) uniquely and easily locates the pen-tip's position on any of the pages of the pad, see [19].

This example shows that it is good engineering to think about designing shapes to have such "self-advertising" properties and this approach could provide us surprisingly efficient and precise metrology devices. This problem was posed by Bruckstein O'Gorman and Orlitsky, at Bell Laboratories, already in 1989, with the aim of designing planar patterns that will serve as location marks, or fiducials on printed circuit boards. The need for location or registration fiducials in printing circuit boards and in processing VLSI devices is quite obvious. When layers of printing and processing are needed in the manufacturing operation, the precision in performing the desired processes in perfect registration with previously processed layers is indeed imperative. The work of [2] showed that there exists an information theoretic bound that limits the location precision for any shape that has an spatial extent of say $A x A$ in pixel-size. Such a shape, when digitized will provide for us about $A^{2}$ meaningful bits of information, via the pattern of black and white pixels in the digitized image. This number of bits can only effectively encode $2^{A^{2}-1}$ different locales, and hence the precision to which we can refine a region one pixel-square in size has a maximal area that must exceed $1 /\left(2^{A^{2}-1}\right)$. If we want balanced $X$ and $Y$ axis precision, we can only locate the pattern to a subpixel precision of $1 /\left[2^{\left(A^{2}-1\right) / 2}\right]$. This is the best precision possible assuming optimal exploitation of the real estate of area $A \mathrm{x} A$, assigned to the location mark. The important issue that was further settled in [2] is the existence of a fiducial pattern that indeed achieves this precision. The pattern is so cute that we exhibit it in Fig. 1.

Looking at this fiducial pattern it becomes obvious what it does. It is indeed a continuous 2D (analog) input that employs the point sampling discretization process to compute its $X$ and $Y$ displacement by providing a binary read-out of the subpixel location of the fiducial within the one pixel to which it can readily be located using the left lowest grid-point (the "rough location" mark) covered by the shape. This leftmost bit of information is also the reason we can only use $A^{2}-1$ bits for subpixel precision, i.e. for cutting the one pixel precision (provided by the "rough location" bit) into locale slices. This process turns the fiducial and the discretization process into a nice analog computer that yields the displacements in the $X$ and $Y$ direction easily, and achieves the highest precision in this task that is possible based on the available data. The analysis provided in [2] goes even further. The optimal fiducials turn out to require highly precise etchings on the VLSI or circuit board devices and hence might be difficult to realize in practice. Hence there is a need to analyze other types of fiducial shapes that achieve suboptimal exploitation of the area, however can provide good location accuracies. For rotational invariance, circularly symmetric shapes turn out to be necessary, and therefore bull-eye fiducials were also proposed in [2] and further analyzed by [7]. 


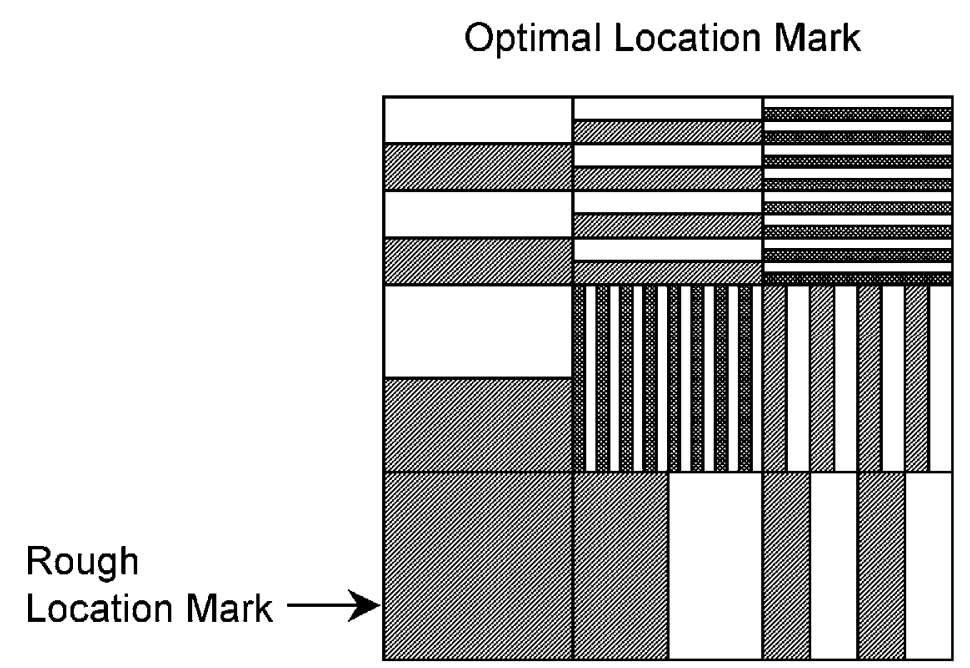

Fig. 1. An optimal 2D fiducial of area $3 \times 3$

The most interesting question that remains to be addressed here is the following: can we invent shapes that provide other metrological measures as easily as the above discussed example advertised its location?

\section{The Importance of Being Gray}

So far we have discussed the case of binary continuous images being pointsampled into matrices of zeros and ones, or Black and White pixels. However the real world is far richer in possibilities and complications.

First of all, point sampling is not a good model of the imaging process as performed by real life cameras. Those carry out, at each sensor level, a weighted integration of the incoming light from the continuous input pattern. This integration happens around each grid point, and the pixel influence region may be assumed circular. The integration yields, at each grid point, values that continuously vary from a lowest value for white (no object) input over the pixel influence region to to highest value that corresponds to having the input object cover the entire area of integration. The result of this integration is then transformed into a discrete value encoded by several bits, via quantization. Therefore even for binary preimages, we get at each grid point a pixel value that is the quantization of a continuous variable proportional to the fraction of the pixel influence region that is covered by the input object.

Furthermore we may also consider the advantages of using non-binary, grayscale of color pre-images. The combination or more realistic sampling and quantization processes with the use or grey levels in preimages open for us a great 
variety of further possibilities. As an example, Kiryati and Bruckstein have analyzed, following a question of Professor Pavlidis, the trade-off between spatial resolution and number of grey levels when the aim is to get as much information as possible on a class of binary pre-images that comprise polygonal shapes. The conclusion of this research was that "Gray Levels Can Improve the Performance of Binary Image Digitizers", see [10]. The paper introduces a measure of digitization-induced ambiguity in recovering the the binary preimage, hence it is quite relevant to metrology under such sampling conditions. It is then shown that, if the sampling grid is sufficiently dense (i.e. the sampling rate is high!) and if the pixels would provide us exact grey-levels rather than quantized values, then error-free reconstruction of the binary pre-image becomes possible. This is not too surprising, however, when the total bit budget for the digitized image representation is limited (i.e. the sampling rate and the quantization depth are related, both being finite) the bit allocation problem that arises shows that the best resource allocation policy is to increase the grey level quantization accuracy as much as possible, once a sufficiently dense spatial sampling resolution has been reached. Therefore once we have a grid dense enough to ensure that all linear borders of the binary input image polygonal shapes can adequately be "seen" in the sampled image, all the remaining bit resources should go towards finer gray level quantization. Professor Pavlidis' question, which prompted this research asked to explain why grey-level fax machines at low resolution yield nicer images than fax machines at higher resolution, even for binary document images. It was clear that some sort of anti-aliasing effect is in place, however [10] showed quantitatively that even in terms of a well-defined metrology error measure, the grey-levels help considerably more than increased spatial resolution.

Imagine next that we allow grey level input images too. In this case we shall certainly have, in conjunction with multilevel quantizations at each pixel much more information for location and various other measurements. A gradual boundary in the input image, or equivalently an area integration sensor providing a quantized multilevel pixel value at each grid-point, will transform the issue of locating a half plane into a problem of locating precisely several parallel digital straight edges, when they are simultaneously sampled. Such richness of detail will certainly dramatically reduce the size of the uncertainty locales, and enable us to design a wealth of improved location and orientation fiducials in the future. For a beginning of work in this direction see the recently completed thesis of Barak Hermesh, titled "Fiducials for Precise Location Estimation".

The conclusion therefore is that gray levels matter, they are good for us! And the last word on these issues certainly has not been said yet.

\section{Concluding Remarks}

Somebody said that every paper and book are autobiographical. This is certainly true of this short paper. It surveys my own research that dealt with digital geometry and metrology issues. As is clear from the list of papers below, my excitement with the interesting topics of research that arise at the interface 
between continuous and discrete geometry is permanent. And I am sure that the attendees of the Discrete Geometry for Computer Imaginary International Conferences all share my excitement with these topics. More on the vast subject of discrete geometry can be found in several books $[4,5,12,14,15]$.

Acknowledgements. Thanks to my past and present students who over the years have taught me so many things on digital geometry and other topics.

Thanks to Professors Theo Pavlidis and Azriel Rosenfeld for their leadership in digital geometry and for their friendship.

Thanks to Yana Katz who made the speedy preparation of this paper possible.

\section{References}

1. R. Bornstein and A.M. Bruckstein, "Finding The Kernel Of Planar Shapes", Pattern Recognition, Vol. 24, No. 11, 1019-1035, 1991.

2. A. M. Bruckstein, L. O'Gorman, and A. Orlitsky, "Design Of Shapes For Precise Image Registration", IEEE Transactions on Information Theory, Vol IT-44/7, 3156-3162, 1998, ( AT\&T Bell Laboratories Technical Memorandum ).

3. A. M. Bruckstein, "Self-Similarity Properties of Digitized Straight Lines", Contemporary Mathematics, Vol. 119, 1-20, 1991.

4. J.-M. Chassery and A. Montanvert, Géométrie Discréte en Analyse d'Images, Hermes, Paris, 1991.

5. L. S. Davis, editor, The Azriel Rosenfeld Book: "Foundations of Image Understanding", University of Maryland, U.S.A., Kluwer, 2001.

6. L. Dorst, "Discrete Straight Line Segments: Parameters, Primitives and Properties", Ph.D. Thesis, Technological University Delft, 1986.

7. A. Efrat and C. Gotsman, "Subpixel Image Registration Using Circular Fiducials", International Journal of Computational Geometry and Applications, Vol.4, 403-422, 1994.

8. D. I. Havelock, "The topology of Locales and Its Effects on Position Uncertainty" IEEE Transaction on Pattern Analysis and Machine Intelligence, Vol. 13, No. 4 380-386, 1991.

9. B. Hermesh, "Fiducials for Precise Location Estimation", Research Thesis, Technion, IIT, Haifa Israel, 2001

10. N. Kiryati and A. M. Bruckstein, "Gray Levels Can Improve the Performance of Binary Image Digitizers" CVGIP: Graphical Models and Image Processing, Vol. 53, No. 1 31-39, 1991.

11. J. Koplowitz, and A. M. Bruckstein, "Design Of Perimeter Estimators For Digitized Planar Shapes", IEEE Trans. on Pattern Analysis and Machine Intelligence, Vol. PAMI-11/6, 611-622, 1989.

12. L. J. Latecki, Discrete Representation of Spatial Objects in Computer Vision, Vol. 11, Kluwer, 1998.

13. M. Lindenbaum and A. M. Bruckstein , "On Recursive, $O(N)$ Partitioning of a Digitized Curve into Digital Straight Segments", IEEE Transaction on Pattern Analysis and Machine Intelligence, Vol. 15, No. 9 949-953, 1993.

14. M. D. McIlroy, "Number Theory in Computer Graphics", The Unreasonable Effectiveness of Number Theory, Proceedings of Symposia in Applied Mathematics, Vol. 46, 105-121, 1992 
15. R. A. Melter, A. Rosenfeld, P. Bhattacharya, editors, "Vision Geometry", Contemporary Mathematics, Vol. 119, AMS, Rhode Island, 1991.

16. D. Proffit and D. Rosen, "Metrication Errors and Coding Efficiency of Chain Coding Schemes for the Representation of Lines and Edges", Computer Graphics Image Processing, Vol. 10, 318-332, 1979.

17. D. Shaked, J. Koplowitz, and A. M. Bruckstein, "Star-Shapedness of Digitized Planar Shapes", Contemporary Mathematics, Vol. 119, 137-158, 1991.

18. K. Voss, Discrete Images, Objects, and Functions in $Z^{n}$, Springer-Verlag, 1991.

19. http://www.anoto.com/technology/anotopen/ 